\title{
Strangulation of the stomach and the transverse colon following laparoscopic esophageal hiatal hernia repair
}

\author{
Jacek Hermann ${ }^{1}$, Tomasz Kościński', Stanisław Malinger ${ }^{1}$, Jacek Szmeja1 ${ }^{1}$, Michał Monkiewicz², Michał Drews ${ }^{1}$ \\ ${ }^{1}$ Department of General, Gastrointestinal and Plastic Surgery, Poznan University of Medical Sciences, Poland \\ 2Department of Imaging Diagnostics, Poznan University of Medical Sciences, Poland
}

Videosurgery Miniinv 2012; 7 (4): 311-314 DOI: 10.5114/wiitm.2011.29251

\begin{abstract}
The authors present a 32-year-old male patient with incarceration of a recurrent esophageal hiatal hernia after laparoscopic repair. A life-threatening strangulation of the stomach and the transverse colon occurred within a few days after the operation. Relapse of hiatal hernias amounts to almost half of early complications characteristic for the laparoscopic approach. General recommendations regarding surgical technique as well as perioperative care have been proposed in order to decrease the risk of relapse. Also, routine contrast radiology on the first or second day following the laparoscopic operation facilitates early diagnosis of relapse of hiatal hernia with emergent reoperation. This may result in decreased morbidity and improved overall outcome of the treatment.
\end{abstract}

Key words: laparoscopic repair of esophageal hiatal hernia, stomach and colon strangulation.

\section{Introduction}

Esophageal hiatal hernias are laparoscopically managed increasingly often due to shorter recovery time and good treatment results, comparable to open procedures. The recurrence rate with this method is reported to vary from $12 \%$ to $55 \%$, depending on the source $[1,2]$. Hernia recurrence constitutes almost half of early characteristic complications of laparoscopic procedures. It may be described as the "Achilles heel" of the laparoscopic approach [3, 4]. The authors present a patient with an incarcerated, recurrent esophageal hiatal hernia, which resulted in a life-threatening strangulation of the stomach and the transverse colon. The patient required surgical intervention.

\section{Case report}

A 32-year-old male patient was admitted to the department on 10.6.2010 due to sliding esophageal hiatal hernia in order to be surgically treated. He reported the symptoms of gastroesophageal reflux refractory to conservative treatment of many years. Apart from that, the patient was generally healthy. On admission day, the physical examination and laboratory tests did not reveal any aberrations. Gastroscopy and esophageal manometry showed hiatal hernia and 24-hour pH-metry confirmed high-grade esophageal reflux. On October 15 laparoscopic repair, accompanied by an anti-reflux procedure, of a small hiatal hernia was performed. Two sutures were placed along the exposed crura of the diaphragm, outside the esophagus, under control of a $60 \mathrm{~F}$ probe inserted into the stomach. After mobilizing the $5 \mathrm{~cm}$ long, abdominal aspect of the esophagus and fundus of the stomach, Nissen fundoplication was performed. On postoperative day zero, the patient vomited multiple times, and after the $1^{\text {st }}$ postoperative day, he reported abdominal pain and swallowing dys-

Address for correspondence:

Jacek Hermann MD, Department of General, Gastrointestinal and Plastic Surgery, Poznan University of Medical Sciences,

49 Przybyszewskiego St, 60-355 Poznan, Poland, phone: +61 869 12 75, fax: +48 61869 16 84, e-mail: jacekhermann@gmail.com 
function, which worsened on the following days. On the $5^{\text {th }}$ postoperative day, dyspnea began to accompany very strong pain ailments. Contrast-induced examination of the upper segment of the alimentary tract revealed early recurrence of the esophageal hiatal hernia. Images made in posteroanterior projection, in a standing position (Figure 1), and left anterior oblique projection in the lying position (Figure 2) confirmed giant esophageal hiatal hernia. Almost the whole stomach was located above the dome of the diaphragm and other structures, such as parts of the transverse colon, connected to the stomach with ligaments, could not be excluded. The dislocated stomach was partially rotated along the long axis of the organ. The hernia pressed against the lower pulmonary lobe and inferior mediastinum. The passage of a contrast medium to the duodenum was inhibited due to the pressure point located at the level of the hiatus and atony of the stomach. The patient was qualified for urgent laparotomy performed on October 21. The incision under costal margins was made in order to gain access to the abdominal cavity. Strangulated esophageal hiatal hernia containing a fragment of the transverse colon and almost the whole stomach was revealed during the process. The viscera

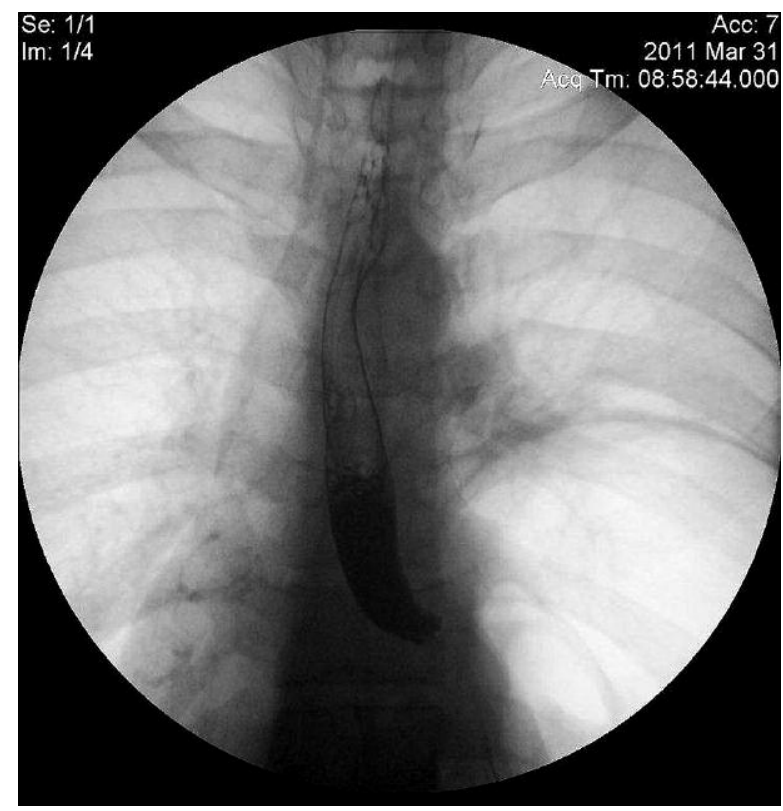

Figure 1. Contrast-enhanced examination of the upper part of the digestive tract. Posteroanterior projection in a standing position. The cardia located above the diaphragm and projected on the distended stomach were pushed back into the peritoneal cavity with difficulty. The blood supply to the stomach and colon did not raise any objections. The crura of the diaphragm became completely separated and the posterior suture was placed for the second time. The postoperative course was complicated with acute respiratory insufficiency. The patient was released on October 26 in a good general condition.

\section{Discussion}

Esophageal hiatal hernia recurrence is totally asymptomatic in over $50 \%$ of patients [3]. Others most commonly present with swallowing dysfunction, abdominal or substernal pain, dyspnea and vomiting of often foamy saliva due to partial or complete obstruction of the dislocated to the mediastinum and rotated stomach. In the worst case scenario the stomach strangulation in the hiatus may lead to malperfusion, gangrene and stomach wall perforation with subsequent mediastinitis and peritonitis. Narrow hernia ring, meaning a small hiatus, increases the risk of incarceration and strangulation of the hernia. In case of the above-mentioned symptoms, contrast-enhanced examination of the upper

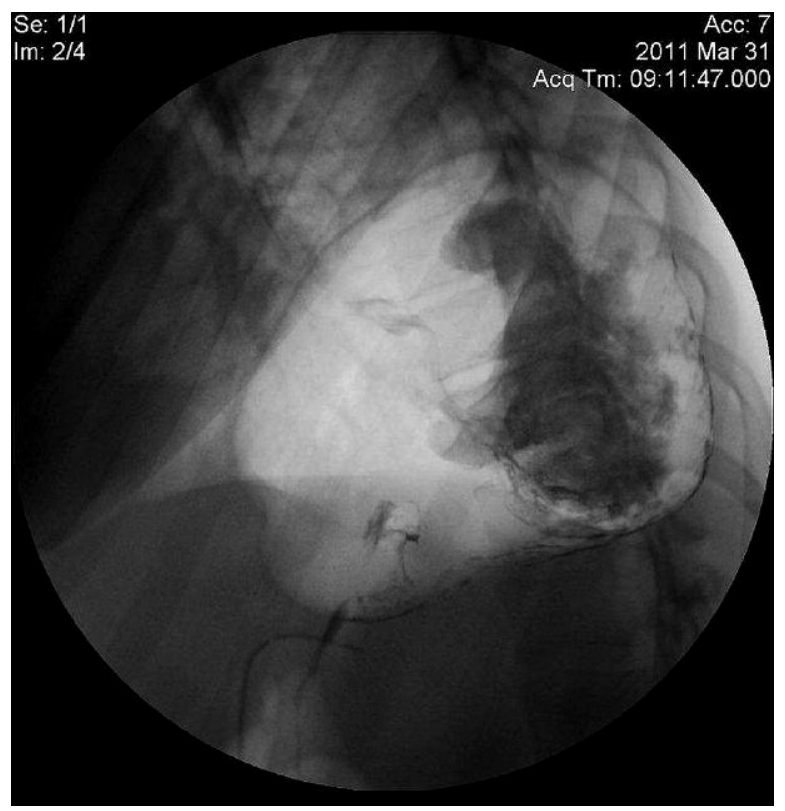

Figure 2. Left anterior oblique projection in the supine position. Giant esophageal hiatal hernia with the distended stomach located in the chest almost entirely. Rotation of the stomach along its long axis 
segment of the alimentary tract should be performed with water soluble contrast media due to the suspected perforation of the stomach wall. When the perforation has been excluded, the principal examination with the use of barium sulfate is performed. It involves careful stomach assessment in the double contrast method [3, 5]. Risk factors typical for the laparoscopic approach, such as interruption of anatomical barriers separating the abdominal cavity from the chest and the increased, in comparison to physiological conditions, pressure gradient between serous cavities of the body, cause further complications. Interruption of the anatomical barriers results from wider esophageal dissection when compared to open surgery. What is more, carbon dioxide filling the peritoneal cavity also separates the esophagus from the surrounding tissues. Next, the dissection of the posterior esophageal wall may result in pleural cavity opening, usually at the left hand side. The stomach is more easily relocated to the pleural cavity then. Nausea and vomiting occur more often after laparoscopic surgery of the esophagogastric junction, probably due to vagus nerve irritation. Additionally, patients more readily use abdominal press and diaphragmatic muscles when coughing and vomiting because of less pain after laparoscopy. In such a case the sutured hiatus may tear and the stomach will more easily get mislocated to the chest [1].

A few general recommendations concerning the surgical technique and perioperative care, which are to decrease the risk of hernia recurrence, have been made. However, they are not based on scientific evidence, so the result of treatment depends mainly on the skills and experience of the surgeon. Crucial importance is attributed to the surgical technique. It consists in dissection of the abdominal portion of the esophagus with exposure of the angle of His, optimal mobilization of the stomach fundus, full removal from the mediastinum and resection of the hernia sac in order to eliminate any tension within the reconstructed tissues. If "short esophagus" is diagnosed, some authors recommend Collis gastroplasty, but according to others such a procedure may increase the recurrence rate of hernia [6, 7]. Proper hiatus management consists in full exposure of crura of the diaphragm and their proper closure behind the esophagus. No possibility of tissue palpation during laparoscopy in order to assess the right diameter of the hiatus prompts the use of a 58-60 F gastric probe. Some authors recommend the use of meshes in case of a large hernia ring, exceeding $5 \mathrm{~cm}$, for strengthening invaluable tissues of the crura. However, the considerable number of complications such as narrowing esophagus and the stomach entrance, esophageal ulceration or mesh migration may discourage their employment [8]. Others, in order to reduce recurrence risk, suggest suturing the fundoplication cuff to the crura of the diaphragm, especially in their posterior aspect, as the stomach most typically migrates behind the esophagus $[9,10]$. The stomach fundus may also be sutured with the abdominal part of the esophagus. This way the angle of His is recreated. Another way is to suture the stomach to the anterior abdominal wall using the Boerem technique $[11,12]$.

Routine contrast-enhanced examination of the upper part of the alimentary tract on the $1^{\text {st }}$ or $2^{\text {nd }}$ postoperative day allows for hernia recurrence early diagnosis and urgent surgical intervention and prevents more serious consequences of the complication $[13,14]$. Administration of antiemetic drugs, antitussives and mild laxatives in the postoperative period may prevent complications due to reduced pressure in the abdominal cavity, similarly to the use of a catheter in patients with urine passage disorder. Also, the patient on the $1^{\text {st }}$ postoperative day should ingest only liquids, and on the following days small portions of food, excluding flatulent dishes [15].

\section{References}

1. Seelig MH, Hinder RA, Klingler PJ, et al. Paraesophageal herniation as a complication following laparoscopic antireflux surgery. J Gastrointest Surg 1999; 3: 95-9.

2. Johnson JM, Carbonell AM, Carmody BJ. Laparoscopic mesh hiatoplasty for paraesophageal hernias and fundoplications: a critical analysis of the available literature. Surg Endosc 2006; 20: 362-6.

3. Abbara S, Kalan MH, Lewicki AM. Intrathoracic stomach revisited. Am J Rad 2003; 181: 403-14.

4. Tarnowski W, Kiciak A, Borycka-Kiciak K, et al. Laparoscopic fundoplication improves oesophageal motility - a prospective study. Videosurgery Miniinv 2011; 6: 73-83.

5. Canon CL, Morgan DE, Einstein DM, et al. Surgical approach to gastroesophageal reflux disease: what the radiologist needs to know. Radigraphics 2005; 25: 1485-99.

6. Agha FP, Trenkner SW, Orringer MB, Vinh PN. The combined Collis gastroplasty-Nissen fundoplication: surgical procedure and radiographic evaluation. Am J Radiol 1985; 145: 729-34.

7. Wallner G, Solecki M, Tarnowski W, et al. Choroba refluksowa przełyku - zalecenia dla praktyki klinicznej. Videosurgery Miniinv 2009; 4 (Suppl 1): 53-61. 
8. Stadlhuber RJ, Sherif AE, Mittal SK, et al. Mesh complications after prosthetic reinforcement of hiatal closure: a 28-case series. Surg Endosc 2009; 23: 1219-26.

9. Gryska PV, Vernon JK. Tension-free repair of hiatal hernia during laparoscopic fundoplication: a ten-year experience. Hernia 2005; 9: 150-5.

10. Wróblewski T, Skalski M, Ziarkiewicz-Wróblewska B, Żurakowski J, Krawczyk M. New antireflux surgical technique in GERD treatment. Videosurgery Miniinv 2007; 2: 139-44.

11. Vrba R, Aujesky R, Vomackova K, et al. Upside-down stomach results of mini-invasive surgical therapy. Videosurgery Miniinv 2011; 6: 231-5.

12. Tsunoda S, Jamiesion GG, Devitt PG, et al. Early reoperation after laparoscopic fundoplication: the importance of routine postoperative contrast studies. World J Surg 2010; 34: 79-84.

13. Poncet G, Maud R, Roman S, Boulez JC. Laparoscopic repair of large hiatal hernia without prosthetic reinforcement: late results and relevance of anterior gastropexy. J Gastrointest Surg 2010; 14: 1910-6.

14. Szyca R, Leksowski K. Cornelia de Lange syndrome - characteristics and laparoscopic treatment modalities of reflux based on own material. Videosurgery Miniinv 2011; 6: 173-7.

15. Balakrishnan S, Singhal T, Grandy-Smith S, et al. Acute transhiatal migration and herniation of fundic wrap following laparo scopic Nissen fundoplication. J Laparoend Adv Surg Tech 2007; 17: 209-12.

Received: 10.04.2011, Revised: 30.12.2011, Accepted: 2.05.2012 